\section{Simplified PCR-Mediated, Linker-Scanning Mutagenesis}

BioTechniques 26:624-626 (April 1999)

Traditional methods of DNA mutagenesis involve the modification of template DNA using chemicals and/or enzymes (2). Deletions, insertions or replacements of specific sequences are accomplished by combining DNA strand cleavage, degradation and subsequent religation. Linker-scanning mutagenesis (LSM), in which adjacent portions of DNA along a defined sequence are individually and sequentially altered, is a technique frequently used to study regulatory regions of expressed genes. These mutagenesis methods can suffer from imprecise mutation localization, tedious and timeconsuming optimizations and the use of enzymatic reactions that are difficult to control. The advent of polymerase chain reaction (PCR) has allowed the streamlining of many previously difficult mutagenesis protocols. Gustin and Burk (1) have previously described a method for generating LSM using PCR. This method involves using four oligonucleotide primers, three PCRs and two ligations for each new mutant. Here, I describe a simplified method for PCR-mediated LSM that generates precisely located replacements of any length on any template DNA. This method involves a single PCR, using a polymerase with proofreading activity and two sequentially positioned oligonucleotide primers on a template plasmid, to generate a linear full-plasmid-length product lacking the desired sequence and the subsequent single ligation of a "linker" oligonucleotide to each of the generated mutant templates. This new technique allows not only the construction of a LSM set of plasmids with identical mutation lengths, but can also be used to generate precisely located mutants with any length of deletion or insertion, while using substantially fewer enzymatic steps and oligonucleotide primers.

This method is demonstrated in the following example by generating a set of LSM mutants of the cytomegalovirus (CMV) enhancer/promoter, a widely used eukaryotic transcriptional control element. In this example, 24 sequential mutants are generated in the CMV promoter, each containing a 25nucleotide (nt) replacement, starting at -675 (relative to the start site of tran-

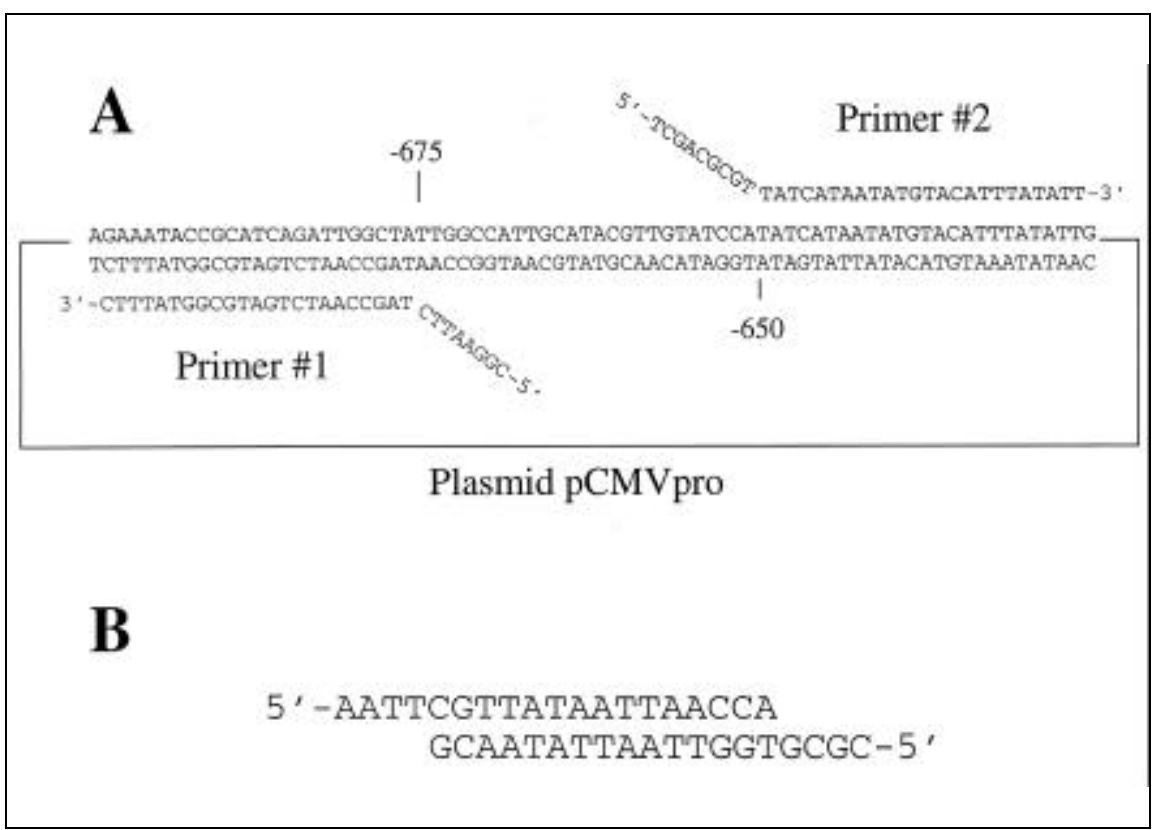

Figure 1. Oligonucleotides used to generate linker-scanning mutant plasmids. (A) Schematic detailing the sequences and locations of the first pair of oligonucleotide primers used in the PCR. (B) Sequence of the double-stranded oligonucleotide linker used in the ligation reaction. scription) and ending at -100 (just upstream of the CCAAT box). As a template for the PCRs, a plasmid containing only the CMV enhancer/promoter, a bacterial origin of replication and an antibiotic resistance gene was constructed (pCMVpro; 3592 nucleotides [nt]). Pairs of opposing strand oligonucleotide primers were synthesized, separated by $25 \mathrm{nt}$ of CMV sequence and designed for use in PCRs to generate a linear product corresponding to the entire plasmid minus that internal 25-nt region (Figure 1A). The primers were 24-nt long and contained, at their $5^{\prime}$ ends, sequences corresponding to restriction enzyme sites absent from the template plasmid (i.e., EcoRI and MluI). Each subsequent pair of primers shifts mutagenesis of the CMV promoter downstream $25 \mathrm{nt}$. Consequently, a set of 24 primer pairs gives complete coverage of this 675-bp CMV sequence. Standard 100- $\mu \mathrm{L}$ PCRs contained $10 \mathrm{ng}$ of plasmid template, $100 \mathrm{pmol}$ of each primer, 200 $\mu \mathrm{mol}$ of each dNTP, $10 \mu \mathrm{L}$ of $10 \times$ reaction buffer [final concentrations of 10 $\mathrm{mM} \mathrm{KCl}, 10 \mathrm{mM}\left(\mathrm{NH}_{4}\right)_{2} \mathrm{SO}_{4}, 20 \mathrm{mM}$ Tris- $\mathrm{HCl}, \mathrm{pH} 8.75,2 \mathrm{mM} \mathrm{MgSO}_{4}$, $0.1 \%$ Triton $^{\circledR} \quad \mathrm{X}-100,100 \mu \mathrm{g} / \mathrm{mL}$ bovine serum albumin (BSA)] and 2.5 U of $P f u$ DNA Polymerase (Stratagene, La Jolla, CA, USA). Reactions consisted of 30 cycles of $94^{\circ} \mathrm{C}$ for $1 \mathrm{~min}, 58^{\circ} \mathrm{C}$ for $2 \mathrm{~min}$ and $72^{\circ} \mathrm{C}$ for $7 \mathrm{~min}$. (Annealing temperatures might need to be optimized due to primer length and composition.) PCR products were then gel-purified and digested with EcoRI and MluI (New England Biolabs, Beverly, MA, USA) overnight. Digested PCR products were ligated to a doublestranded linker oligonucleotide (Figure 1B) designed with EcoRI and MluI compatible ends. This linker is of an appropriate length, such that the final ligated product restores the same size as the template plasmid. The internal linker sequence can be random or can be used to introduce specific sites into the template; in this case, it contained a consensus $\mathrm{A} / \mathrm{T}$ rich transcription-factor binding site. Transformations and subsequent restriction endonuclease analysis of miniprep DNA can be used to quickly identify correct new clones. These mutant promoters can now be transferred to reporter gene constructs 


\section{Benchmarks}

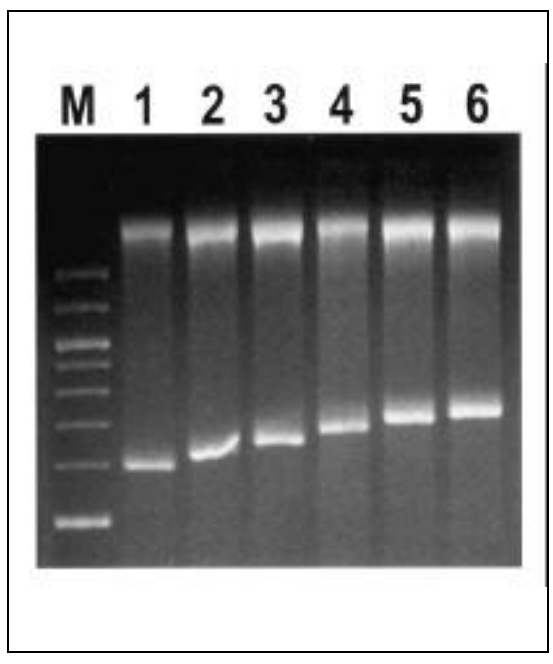

Figure 2. Restriction digest of LSM plasmids detailing shifted location of mutant linker. The first six plasmids in the CMV-LSM series were digested with $\mathrm{XhoI}$ and $M l u \mathrm{I}$ and electrophoresed on a $2 \%$ agarose TBE gel for $1.5 \mathrm{~h}$ at $100 \mathrm{~V}$. Lane M, 100-bp marker (New England Biolabs); lanes 1-6, plasmids 1-6. Expected fragment sizes are $608,633,658,683,708$ and 733 bp, respectively.

for functional analysis. Mutagenesis to the small promoter-only containing plasmid is preferred to that of the reporter gene construct, because amplification of a smaller plasmid is faster and more efficient and removes the slight possibility of introducing random mutations to the reporter gene. The transferred mutant region can be sequenced to confirm that no random mutations were introduced during amplification.

Each new plasmid is the same size as the parental plasmid and differs only in the sequential 25-nt replacement. Figure 2 details a restriction digest of the first six plasmids in the series and illustrates the shifting mutation (XhoI cuts upstream of the mutations; $M l u \mathrm{I}$ is contained in the mutations). In this example, linker length was specified to maintain the relative distances and topology between other sequences in the enhancer/promoter, but it is not necessary to do so. Thus, the power of this technique lies in the wide variety of mutants that can be generated. Using only one template, a relatively small series of primers and as many linkers as desired, literally hundreds of mutants, differing in size, location and content, can be generated with a single set of PCRs and ligations.

\section{REFERENCES}

1.Gustin, K.E. and R.D. Burk. 1993. A rapid method for generating linker scanning mutants utilizing PCR. BioTechniques 14:22-24.

2.Sambrook, J., E.F. Fritsch and T. Maniatis. 1989. Molecular Cloning: A Laboratory Manual, 2nd ed. CSH Laboratory Press, Cold Spring Harbor, NY.

Address correspondence to Dr. Kerry M. Barnhart, Vical, Inc., 9373 Towne Centre Drive, Suite 100, San Diego, CA 92121, USA. Internet: kbarnhart@vical.com

Received 15 September 1998; accepted 8 December 1998.

Kerry M. Barnhart

Vical, Inc.

San Diego, CA, USA

\section{Increased Efficiency of Arbitrarily Primed PCR by Prolonged Ramp Times}

\author{
BioTechniques 26:626-630 (April 1999)
}

Arbitrary primers are widely used in several applications of the polymerase chain reaction (PCR), including differential display PCR (6) and the random amplified polymorphic DNA (RAPD) (10). Whereas the first method has been demonstrated to be a powerful tool for the identification of differentially expressed genes, RAPD is often performed for analyzing genetic variabilities by generating a genomic fingerprint. However, due to the low annealing temperatures required for short, unoptimized primers, both resolution and reproducibility of arbitrarily primed PCRs are often low (2,3). To overcome this problem, several improvements have been suggested concerning $(i)$ the temperature profile, (ii) the quality of the DNA template, (iii) the number and length of PCR cycles and (iv) the technical equipment $(1,7)$. With respect to the ramp time between the annealing and the extension temperature, different observations have been made. Lawrence et al. (5) reported a benefit for the reproducibility of RAPD by performing a 1-min ramp, whereas Benter et al. (1) observed no general influence on the reproducibility of RAPD by prolonged ramp times but an increase in number and yield of the DNA bands obtained. A similiar effect on the band pattern obtained was found for RAPD by introducing a transition interval between melting and annealing temperatures (9). Small variations in ramp time (9-40 s) had little or no effect on the resolution and reproducibility of differential display PCR (8).

Here, we demonstrate that drastically prolonged ramp times between the annealing and the extension temperatures (up to several minutes) lead to a profound increase in number and yield of the DNA bands obtained in both methods. The benefit of our observation for differential display PCR lies in the improved detection of low-abundance mRNA species that could other- 